\title{
Cost-effectiveness of routine measuring of serum drug concentrations and anti-drug antibodies in treatment of rheumatoid arthritis patients with TNF- $\alpha$ blockers
}

\author{
This article was published in the following Dove Press journal: \\ Biologics:Targets and Therapy \\ I April 2016 \\ Number of times this article has been viewed
}

Juha Laine'

T Sakari Jokiranta ${ }^{2,3}$

Kari K Eklund ${ }^{4,5}$

Merja Väkeväinen'

Kari Puolakka ${ }^{6}$

'Pfizer Oy, Helsinki, ${ }^{2}$ United Medix Laboratories Ltd, Espoo, ${ }^{3}$ Research Programs Unit, Immunobiology, ${ }^{4}$ Department of Rheumatology, University of Helsinki, ${ }^{5} \mathrm{Helsinki}$ University Central Hospital, Helsinki, ${ }^{6}$ Department of Medicine, South Karelia, Finland
Correspondence: Juha Laine

Pfizer Oy, Tietokuja 4, 00330 Helsinki,

Finland

Tel +3589430040

Email juha.laine@pfizer.com

\begin{abstract}
Monitoring of anti-drug antibodies (ADAbs) or serum concentrations of biologicals in treatment of rheumatoid arthritis could provide an explanation for a loss of efficacy and help in the choice of subsequent medication. Current clinical practices do not generally include such monitoring of tumor necrosis factor (TNF)- $\alpha$ blockers on a routine basis. The main aims of this study were to estimate the probabilities of optimal and nonoptimal treatment decisions if infliximab or adalimumab drug trough level (DL) and ADAbs are tested or not in rheumatoid arthritis, and to model cost-effectiveness of performing such monitoring on a routine basis. Data on DLs and ADAbs concentrations were obtained in Finland from clinically requested monitoring analyses of 486 and 1,137 samples from patients on adalimumab and infliximab, respectively. DL was within the target range in $42 \%$ of samples from adalimumab- and $50.4 \%$ of infliximab-treated patients. ADAbs were detected in approximately $20 \%$ and $13.5 \%$ of samples from adalimumab- and infliximab-treated patients, respectively. ADAbs were found in $52.3 \%$ and $41.3 \%$ of those with low adalimumab or infliximab DLs, respectively. The monitoring data were incorporated into probabilities for making the optimal treatment decision. Economic impact of clinical decision-making was modeled in a short-term (3-6 months) scenario with 100 hypothetical patients. In the model, the combined measurement of DLs and ADAbs was cost-saving compared to the nontesting scenario when the monitoring results affected the treatment decision in at least $2-5$ of 100 patients, a proportion which is easily exceeded in real-life clinical practice. This study indicates that routine monitoring of drug level and ADAbs is cost-beneficial in clinical practice, thereby improving the decision-making process in using TNF- $\alpha$ blockers.
\end{abstract}

Keywords: anti-TNF drugs, anti-drug antibodies, trough level measurement

\section{Background}

Biological pharmaceuticals, especially the tumor necrosis factor (TNF)- $\alpha$ blockers, are widely used for the treatment of chronic inflammatory diseases such as rheumatoid arthritis (RA), psoriasis, and Crohn's disease. However, more than one-third of these patients either do not respond to the treatment or lose their initial response within a few years. ${ }^{1,2}$ The failure of TNF- $\alpha$ blockers has increasingly been attributed to generation of anti-drug antibodies (ADAbs), as several studies have demonstrated association between the emergence of ADAbs, low serum drug concentration, and impaired clinical efficacy. ${ }^{3-7}$

Current clinical practices in Finland do not generally include routine monitoring of ADAbs or serum concentrations of any biological, although this information could provide an explanation for partial or complete loss of efficacy and help in the choice of 
subsequent medication. In some other countries, monitoring is likely to become part of routine care of patients with inflammatory bowel diseases, especially in the context of loss of response. On the basis of the drug levels and ADAbs, Vincent et $\mathrm{al}^{1}$ have proposed a model algorithm for helping take a rational decision between switching the ongoing biological drug to one with the same or another mode-of-action and changing the dose of the ongoing drug.

The rationale of monitoring drug level and ADAbs in TNF- $\alpha$-treated patients is based on multiple reasons such as lost efficacy, primary non-responsiveness, and workup of adverse events. When a patient has become clearly immunized against a biological, as indicated by a high level of ADAbs in serum, that drug is destined to fail. ${ }^{3}$ If the immunization is not noticed, the treatment is often continued and the drug dose is further increased, apparently resulting in needless costs. However, with some patients who have ADAbs, addition of methotrexate or increase in the drug dose may result in improved drug trough level (DL). In some patients with abrupt loss of efficacy of one biological due to ADAbs, the whole mode-of-action may be inadequately abandoned. On the other hand, if a drug concentration lower than the target range drug concentration leads to the loss of efficacy in the absence of ADAbs, a dose increase could result in improved clinical efficacy, although drug costs are simultaneously increased, and increasing the dose of TNF- $\alpha$ blocker may not necessarily provide additional efficacy. ${ }^{8}$ To conclude, systematic monitoring of drug concentrations and ADAbs could be potentially beneficial and economically justified, especially given the high costs of biopharmaceuticals and the complexity of clinical decision-making.

Several real-life data studies have shown that costs of different TNF- $\alpha$ blockers are not equal, for instance, because dose escalations are more frequent with some of them compared to others. This is obviously because of the varying tendency to generate ADAbs. ${ }^{9-14}$ The economic impact of drug level and ADAb monitoring is, however, not studied in the real-life setting. The aim of this study was to estimate the probability of optimal and nonoptimal treatment decisions if the drug levels and ADAbs of the two most used monoclonal antibody drugs in RA - infliximab and adalimumab - are tested or not, and based on this information, to explore the economic implications of the routine testing.

\section{Methods}

\section{Laboratory data}

Real-life data on DLs and ADAb concentrations of infliximab and adalimumab formed the database used in this study.
The data were obtained from the clinical sample registry of United Medix Laboratories Ltd in Helsinki, Finland. All the samples included in the database had been sent to the laboratory on a clinical basis, ie, none of the samples were from clinical studies. The DLs had been analyzed using enzymelinked immunosorbent assay, and the ADAb was analyzed by radioimmunoassay. ${ }^{3,15,16}$ All the ADAb measurements and the adalimumab DLs had been outsourced to Sanquin Diagnostic Services, Amsterdam, the Netherlands, while approximately half of the infliximab DLs were analyzed by the United Medix Laboratories using ProMonitor test kit (Progenica, Derio, Spain).

The use of samples in the study has been approved by the coordinating ethical review board of the hospital district of Helsinki and Uusimaa (ref no 406/13/03/00/15).

\section{Population stratification according to $D L$ and ADAb}

The "optimal level" or target level of infliximab was defined as $\geq 2 \mu \mathrm{g} / \mathrm{mL}$ and that of adalimumab as $\geq 5 \mu \mathrm{g} / \mathrm{mL}$, while the "low level" was defined as $<2$ and $<5 \mu \mathrm{g} / \mathrm{mL}$, respectively. The positivity in the ADAb test was defined as $\geq 12 \mathrm{U} / \mathrm{mL}$ for both infliximab and adalimumab ADAbs. The patient population stratification in the analysis was based on cross tabulation of the real-life DL and ADAb results.

For adalimumab, the lower limit of the target range $(5 \mu \mathrm{g} / \mathrm{mL})$ was taken from the study with RA patients and follows the average used in a previous study. ${ }^{17}$ For infliximab, we chose $2 \mu \mathrm{g} / \mathrm{mL}$ as the lower limit in order to not exaggerate the number of patients with low drug level.

\section{Likelihood of changes in drug use}

The data on DLs and ADAbs were incorporated into transition probabilities and states in a Markov model for determining the optimal treatment in the following 6-month cycle. States in the Markov model were 1) the first TNF- $\alpha$ blocker, 2) the second biological (TNF- $\alpha$ blocker or non-TNF drug), and 3) quitting biologicals. Any switch to a third biological was not taken into account. Transition probabilities related to the nonoptimal treatment response (nonresponse) in the absence of knowledge of DL and ADAb values were derived from previous literature for both the first-line treatment (European League Against Rheumatism [EULAR] response at 6 and 12 months) and the second-line treatment. ${ }^{18,19}$ In the case of EULAR nonresponse, the stratified data on DLs and ADAbs were then used to determine the optimal individualized treatment decision distribution according to a simplified treatment decision algorithm. ${ }^{1}$ The Markov model was used 
to illustrate drug survival and expected medication in the population as a result of optimal switches between the drugs within a 3-year scenario.

\section{Cost estimation}

In the second stage, economic impact of clinical decisionmaking was modeled in a short-term (3-6 months) scenario with 100 hypothetical patients. A wrong clinical decision was defined to lead to ineffective treatment for at least 3-6 months, according to the typical follow-up visit frequencies of RA patients treated with biologicals in Finland. The patient's condition was assumed to be still suboptimal at the next visit, thereby leading to the need for further treatment modifications.

The cost of 1-month nonoptimal treatment was estimated to be approximately $€ 1,471$ in total, including drug cost ( $€ 1,140$, the drug cost estimate per month of subcutaneous TNF- $\alpha$ blocker without value added tax), travel and lost working and leisure time costs for a laboratory visit (€17.4), costs of the possible standard safety-related laboratory tests (€6.8), travel and lost working and leisure time cost for a follow-up visit to an outpatient specialist clinic (€66.6), and specialist visit (€240.6). Long-term efficacy-related costs were not modeled. Cost of resources was valued according to the national unit costs converted to the value of year $2013 .^{20}$

\section{Results}

\section{Distribution of infliximab and adalimumab DLs and ADAb results in Finland}

DLs in $42.0 \%$ of samples were within the "optimal range" (5-10 $\mu \mathrm{g} / \mathrm{mL}$ or higher), while $35.8 \%$ had a "low level" ("DL optimal" and "DL low" in Figure 1A). Approximately $20 \%$ of adalimumab users had any ADAbs ( $\geq 12 \mathrm{AU} / \mathrm{mL})$ (sum of "ADAb+" in Figure 1A). In the dataset, approximately $15 \%$ reached levels more than $30 \mathrm{AU} / \mathrm{mL}$. Of the samples with optimal DL, 97.1\% (198/204) contained no adalimumab ADAbs. On the contrary, of the 174 samples with low adalimumab level, 52.3\% were positive for ADAbs (not reported in Figure 1).

In the database, a total of 1,137 simultaneous infliximab DL and ADAb results were found; $50.4 \%$ of the DLs were within the "optimal range" $(2-10 \mu \mathrm{g} / \mathrm{mL}$ or higher), while $32.4 \%$ were below this "low level" ("DL optimal" and "DL low" in Figure 1B). Approximately $13.5 \%$ of patients had antiinfliximab antibodies (sum of "ADAb+" in Figure 1B).

In parallel with adalimumab, $99.8 \%$ of the samples with optimal DL were negative for infliximab ADAbs, while
ADAbs were found in $41.3 \%$ of those with low DL (not reported in Figure 1).

The dataset also indicated that the distributions of DLs and ADAbs were skewed, and the association between these was nonlinear. The distributions were actually somewhat exponential. The joint distributions of DLs and ADAbs were also different for adalimumab and infliximab, and so the relationship between DL and ADAb is not fully consistent for adalimumab and infliximab.

\section{Decision-making model and expected drug modifications}

The algorithm by Vincent et $\mathrm{al}^{1}$ was used to build up a Markov model. However, the option for optimal DL with detectable ADAbs was removed because only a few patients were classified in this group in our laboratory dataset for adalimumab and for infliximab. Moreover, to avoid overestimation of the economic impact of testing, dose increase was not allowed. This also reflects the real-life situation in Finland. National reimbursement statistics in Finland indicate that dose increase is not very widely used (unpublished data from Social Insurance Institutions). Instead, the patients who would have needed dose escalation (low trough level and no ADAb) were treated as those switching to another TNF- $\alpha$ blocker or a non-TNF biological. When this simplified algorithm was used in conjunction with our laboratory dataset, the Markov model predicted that within the following 3 years, $40 \%$ of those on adalimumab (Figure 2A) and $50 \%$ of the patients on infliximab (Figure 2B) would need drug treatment modifications. We assumed that a small fraction of patients totally quit using biologicals.

\section{Cost analyses}

Patient distributions according to DL and ADAb database (Figure 1) and Markov model (Figure 2) indicate that lack of information about serum drug levels and ADAbs can lead to nonoptimal clinical treatment decisions. These apparently result in use of TNF- $\alpha$ blockers or non-TNF drugs (or escalated dose) without satisfactory response and lead to unnecessary costs. To estimate the costs and savings related to routine monitoring of drug DLs and ADAbs, we built up a hypothetical cohort $(\mathrm{N}=100)$ of patients from whom both serum DLs and ADAbs were measured. The cost of testing was estimated to amount maximally to $€ 20,000$ ( $€ 200 \times 100$ patients). The costs incurring from nonoptimal treatment decision upon secondary loss of response (€1,471/month) accumulate with the pace of clinical follow-up visits, and 

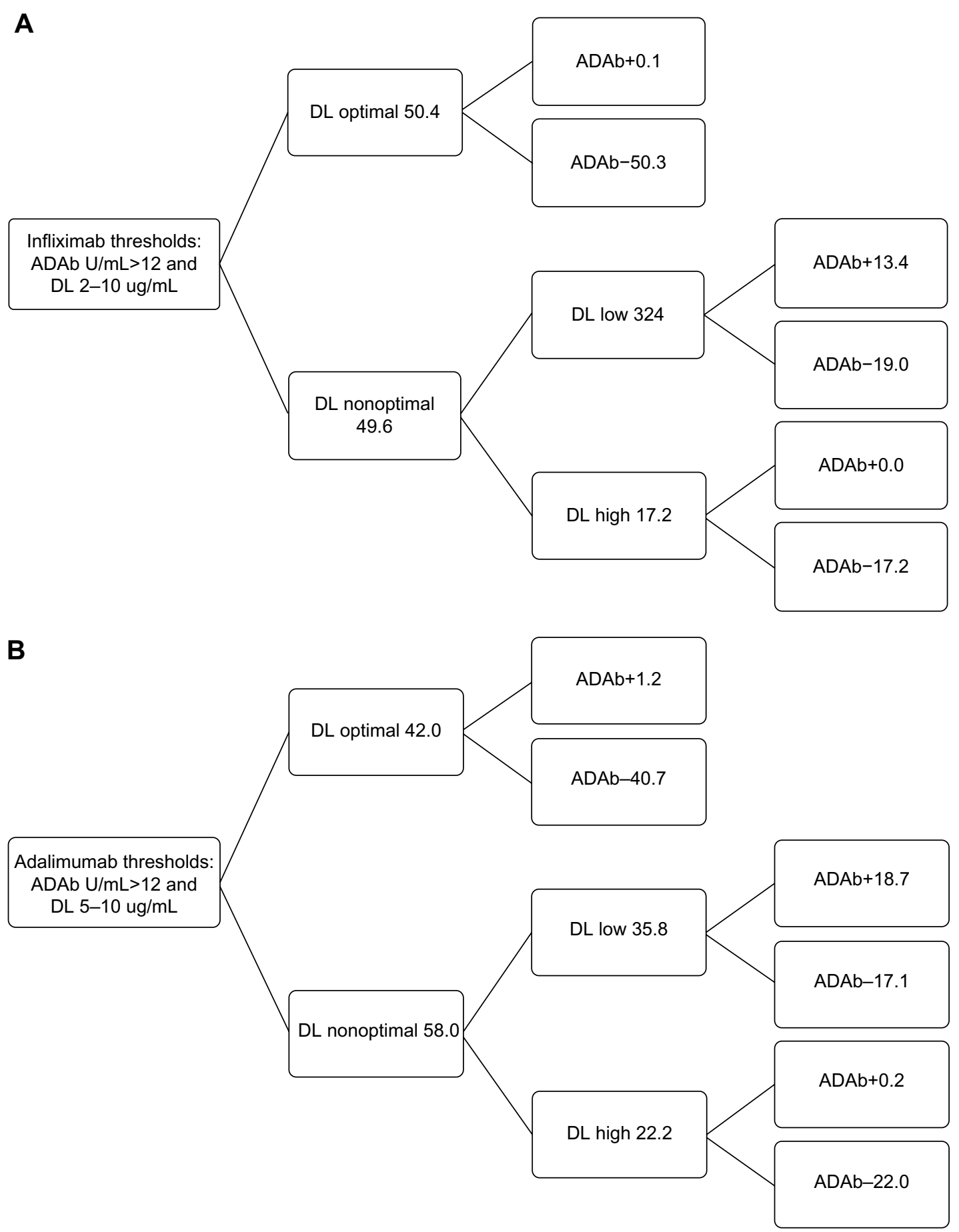

Figure I Stratification of a hypothetical patient cohort on the basis of the real-life monitoring result database of this study.

Notes: (A) Expected stratification of 100 patients on adalimumab treatment. (B) Expected stratification of 100 patients on infliximab treatment.

Abbreviations: DL, drug trough level; $A D A b+$, positive anti-drug antibody analysis result; ADAb-, negative anti-drug antibody analysis result.

therefore we varied visit frequencies (3, 4.5, and 6 months) in the analyses.

Figure 3 illustrates the inverted correlations between the incurred costs/savings of systematic testing and the number of nonoptimal treatment decisions in the cohort of 100 patients. Break-even points lie between two and five out of 100 decisions.
The ineffective treatment of five patients, only for 3 months or 2.5 patients for 6 months is estimated to cost approximately $€ 22,065$ ( $€ 1,471 \times 3$ months $\times 5$ patients). Consequently, the routine measurement of both DLs and ADAbs can be cost-saving compared to the nontesting scenario, when only $2.5-5$ of 100 patients were treated nonoptimally for 3-6 months. 
A

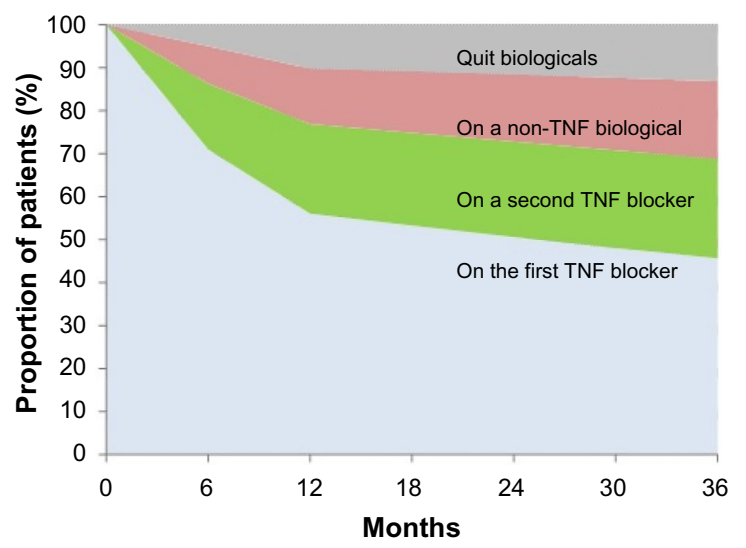

B

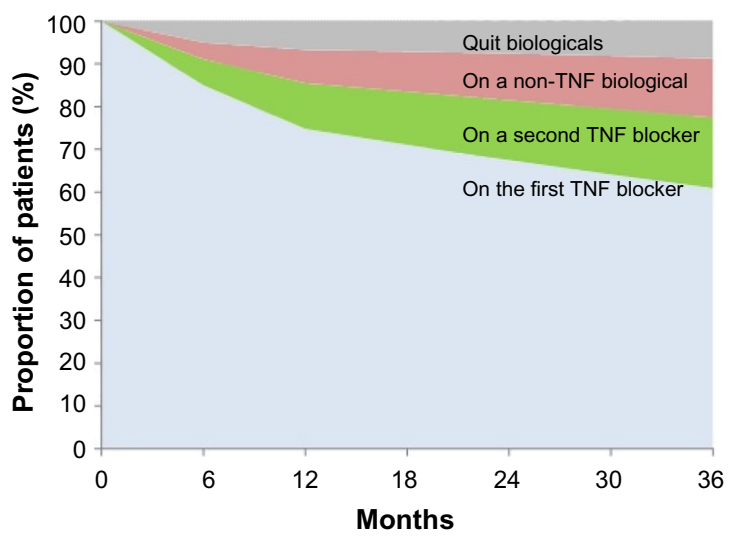

Figure 2 Markov model of the proportion of patients on adalimumab (A) or infliximab (B) within the modeled 3-year follow-up. Abbreviation: TNF, tumor necrosis factor.

\section{Discussion}

Adoption of biological drugs has not only improved treatment results in many chronic inflammatory diseases but has also led to greatly increased drug costs. Because of the budget impact of using biologicals, cost-effectiveness should be a major aim. Treatment response needs to be monitored clinically and medication discontinued or switched, or in rare cases, the dose escalated, in case of insufficient efficacy. Considering the anti-TNF monoclonal antibody drugs, their immunogenicity potentially leading to generation of ADAbs may result in loss of treatment response.
Our study indicates that the probability of nonoptimal drug treatment decision is noteworthy among the Finnish patients using infliximab and adalimumab if the data on individual DLs and ADAbs are not available. Even at very low probability of nonoptimal decision, the systematic measurement of DLs and ADAbs seems to be cost-saving. The main reason for this is that the testing cost of $€ 200$ (combined DL and ADAb) is small compared to the daily treatment costs with TNF or non-TNF biologicals.

Based on the data presented in the analysis, a stepwise approach could also be suggested: DL is tested for all

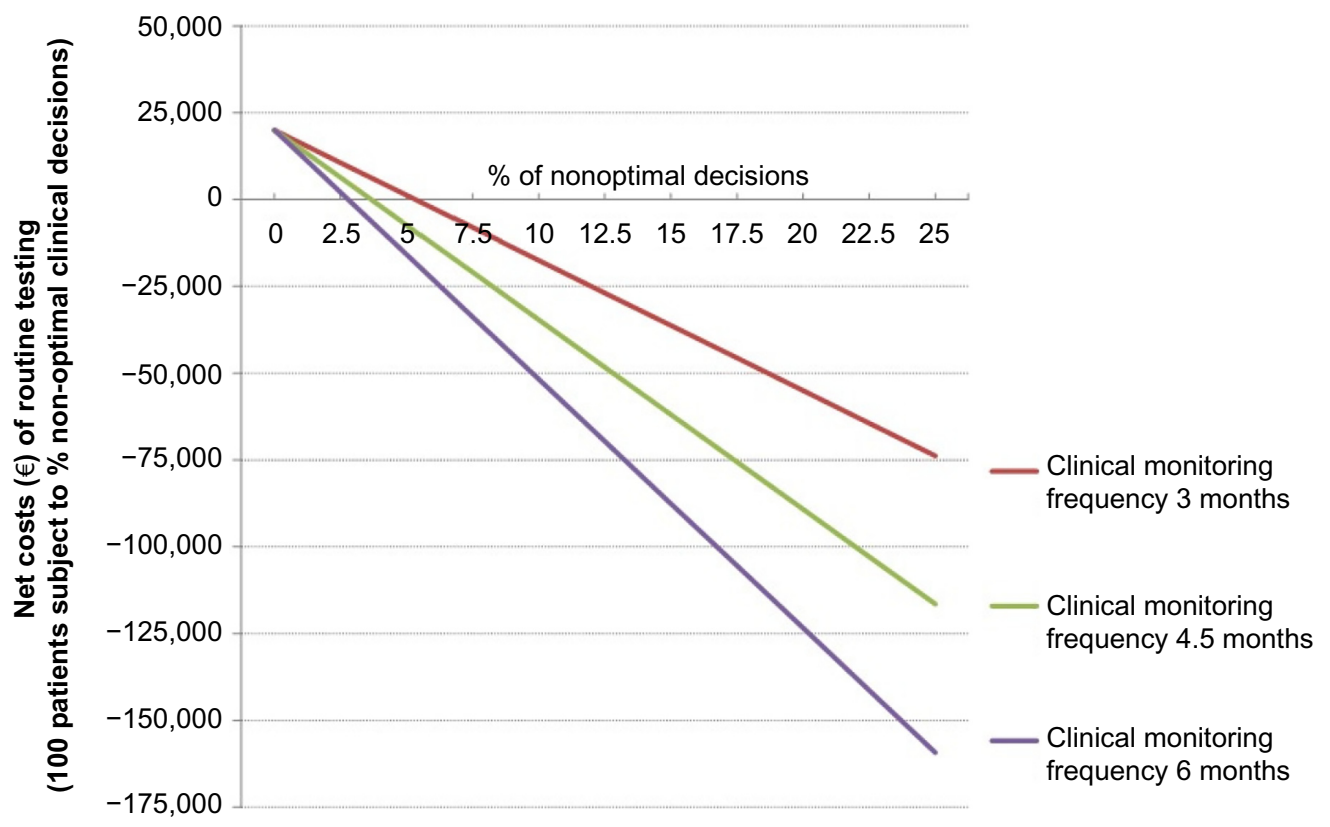

Figure 3 Costs or savings of systematic testing of drug trough level and ADAb.

Notes: The $X$-axis denotes the percentage of patient cases in which a nonoptimal decision is made in the absence of individual DL and ADAb data. The $Y$-axis shows the net economic effect of routine testing and avoidance of nonoptimal treatment decisions. The three colored lines indicate the net costs or savings depending on the frequency of clinical follow-up visits (3-, 4.5-, or 6-month intervals) of the patients on TNF- $\alpha$ blockers.

Abbreviations: DL, drug trough level; ADAb, anti-drug antibody; TNF, tumor necrosis factor. 
patients, but ADAbs are tested for only when DL is low. This saves testing costs but carries a risk for nonoptimal decision. This holds true especially for adalimumab: a small fraction $(1.2 \%)$ of patients had ADAbs even when DL was optimal. The ADAb levels, however, were low (12-30 AU/mL).

Our results are conservative as dose escalation was not allowed in the model. One important question is how to value utilization of nonoptimal drug. We took only short-term health care costs into account, but nonoptimal treatment may have long-term and wider societal consequences, too. These include, for instance, costs attributed to families, employers, and tax payers as well as an impact on health benefits.

The real-life laboratory data on simultaneous DLs and ADAbs from a relatively large number of patients enabled the analyses. Our dataset is larger than the corresponding datasets from other countries and is the first one with real-life data. The main limitation of our analysis is that the laboratory data also contained sera from patients with indications for TNF inhibitors other than RA and that the reasons for testing are likely to be various. Our DL and ADAb data were, however, relatively similar to most of the previously published data from controlled trials with RA patients, thus indicating that the aforementioned limitations are unlikely to have a major impact on the results regarding the probability of nonoptimal drug treatment decision.

Some variability of ADAb positivity can be seen in the literature, and this is likely to be caused by variable cutoff of ADAb positivity. ${ }^{1}$ While infliximab ADAbs are found only in those patients with low drug level, indicating clinical significance, some patients with low level of adalimumab ADAbs have also relatively high drug level. Because of this, currently in Finland, interpretation of clinically significant adalimumab ADAb positivity starts from $30 \mathrm{U} / \mathrm{mL}$, not $12 \mathrm{U} / \mathrm{mL}$. Unnecessarily high drug concentrations might allow lower drug dose or longer interval. ${ }^{21}$ It should also be mentioned that various ADAb tests are available and that only one was used in this study. In order to avoid hardly justifiable change of treatment in the patients with DL within the target range and low positive adalimumab ADAb level, it has been suggested that the interpretation of DL result should be primary, and ADAb results are considered clinically relevant only in case DL is suboptimal.

There are a few potential reasons for low level of drug in the absence of ADAbs. First, it is known that there is considerable individual variation in metabolizing biological drugs. Second, poor compliance can have a dramatic effect on drug levels in the case of adalimumab. Third, since the $\mathrm{ADAb}$ tests measure antibodies against only the Fab2 fragment of the drug, it remains possible (although not shown) that some patients with antibodies against Fc part of the drug would have low drug levels despite the negative ADAb test result.

On the basis of the economic analysis, routine monitoring of TNF-inhibitor DL and ADAb should be included in clinical practice. The traditional decision-making based on physicians' experience, tolerance of methotrexate, patients' preferences, etc, can be improved by these tests. This could result not only in clinical benefit for the patient by finding more quickly the optimal drug for each patient in the case of loss of efficacy to one, but also in more cost-effective use of biologicals per se. In future, the results of this study need to be verified by a prospective study in which the incurred costs of monitoring, drugs, and clinical visits are followed in a large cohort of patients with RA.

\section{Disclosure}

Juha Laine is an employee of Pfizer Finland. T Sakari Jokiranta is employed as Medical Director, United Medix Laboratories; he has received honoraria for lecturing and/ or consultation fees from the following pharmaceutical companies: Abbvie, Alexion Pharmaceuticals, MSD, Pfizer, Roche, Takeda. Kari K Eklund has received research support from Pfizer Ltd. Merja Väkeväinen is an employee of Pfizer Finland. Kari Puolakka is employed as senior rheumatologist, South Karelia Central Hospital; he has received honoraria for lecturing and/or consultation fees from the following pharmaceutical companies: Abbvie, BMS, MSD, Pfizer, Roche, and UCB. The authors report no other conflicts of interest in this work.

\section{References}

1. Vincent FB, Morand EF, Murphy K, Mackay F, Mariette X, Marcelli C. Antidrug antibodies (ADAb) to tumour necrosis factor (TNF)-specific neutralizing agents in chronic inflammatory diseases: a real issue, a clinical perspective. Ann Rheum Dis. 2013;72:165-178.

2. Virkki LM, Valleala H, Takakubo Y, et al. Outcomes of switching antiTNF drugs in rheumatoid arthritis - a study based on observational data from the Finnish Register of Biological Treatment (ROB-FIN). Clin Rheumatol. 2011;30:1447-1454.

3. Bartelds GM, Krieckaert CL, Nurmohamed MT, et al. Development of antidrug antibodies against adalimumab and association with disease activity and treatment failure during long-term follow-up. JAMA. 2011;305:1460-1468.

4. Plasencia C, Pascual-Salcedo D, Nuño L, et al. Influence of immunogenicity on the efficacy of longterm treatment of spondyloarthritis with infliximab. Ann Rheum Dis. 2012;71:1955-1960.

5. Kneepkens EL, Wei JC, Nurmohamed MT, et al. Immunogenicity, adalimumab levels and clinical response in ankylosing spondylitis patients during 24 weeks of follow-up. Ann Rheum Dis. 2015;74:396-401.

6. Menting SP, Sitaram AS, van der Stok HM, de Rie MA, Hooft L, Spuls PI. Drug survival not significantly different between biologics in patients with psoriasis vulgaris: a single center database analysis. Br J Dermatol. 2014;171:875-883. 
7. Garcês S, Demengeot J, Benito-Garcia E. The immunogenicity of anti-TNF therapy in immune-mediated inflammatory diseases: a systematic review of the literature with a meta-analysis. Ann Rheum Dis. 2013;72:1947-1955.

8. Aaltonen K, Virkki L, Malmivaara A, et al. Systematic review and metaanalysis of the efficacy and safety of existing TNF blocking agents in treatment of rheumatoid arthritis. PLoS One. 2012;7:e30275.

9. Ariza R, Navarro-Sarabia F, Hernández-Cruz B, Rodríguez-Arboleya L, Navarro-Compán V, Toyos J. Dose escalation of the anti-TNF- $\alpha$ agents in patients with rheumatoid arthritis. A systematic review. Rheumatology (Oxford). 2007;46:529-532.

10. Kristensen L, Geborek P, Saxne T. Dose escalation of infliximab therapy in arthritis patients is related to diagnosis and concomitant methotrexate treatment: observational results from the South Swedish Arthritis Treatment Group register. Rheumatology (Oxford). 2009;48:243-245.

11. Blom M, Kievit W, Kuper HH, et al. Frequency and effectiveness of dose increase of adalimumab, etanercept, and infliximab in daily clinical practice. Arthritis Care Res (Hoboken). 2010;62:1335-1341.

12. Kievit W, Fransen J, Adang EM, et al. Long-term effectiveness and safety of TNF-blocking agents in daily clinical practice: results from the Dutch Rheumatoid Arthritis Monitoring register. Rheumatology (Oxford). 2011;50:196-203.

13. Moots RJ, Haraoui B, Matucci-Cerinic M, et al. Differences in biologic dose-escalation, non-biologic and steroid intensification among three anti-TNF agents: evidence from clinical practice. Clin Exp Rheumatol. 2011;29:26-34.

14. Moots RJ, Mays R, Stephens J, Tarallo M. Burden of dose escalation with tumour necrosis factor inhibitors in rheumatoid arthritis: a systematic review of frequency and costs. Clin Exp Rheumatol. 2015;33(5):737-745.
15. Wolbink GJ, Vis M, Lems W, et al. Development of antiinfliximab antibodies and relationship to clinical response in patients with rheumatoid arthritis. Arthritis Rheum. 2006;54:711-715.

16. van Schouwenburg PA, van de Stadt LA, de Jong RN, et al. Adalimumab elicits a restricted anti-idiotypic antibody response in autoimmune patients resulting in functional neutralisation. Ann Rheum Dis. 2013;72:104-109.

17. Pouw MF, Krieckaert CL, Nurmohamed MT, et al. Key findings towards optimising adalimumab treatment: the concentration-effect curve. Ann Rheum Dis. 2015;74(3):513-518.

18. Hetland ML, Christensen IJ, Tarp U, et al. Direct comparison of treatment responses, remission rates, and drug adherence in patients with rheumatoid arthritis treated with adalimumab, etanercept, or infliximab: results from eight years of surveillance of clinical practice in the nationwide Danish DANBIO registry. Arthritis Rheum. 2010;62:22-32.

19. Herbild L. Behandling med biologiske laegemidler blandt gigtpatienter: En modelbaseret cost-effectiveness analyse af antistofmonitorering [A model-based cost-effectiveness analysis of the measurement of antibodies]. NOTAT/Projekt 3225. København, Denmark: Dansk Sundhedsinstitut; 2012. Available from: http://www.kora.dk/media/272051/ dsi-3225.pdf. Accessed August 26, 2015

20. Hujanen T, Kapiainen S, Tuominen U, Pekurinen M. Terveydenhuollon yksikkökustannukset Suomessa vuonna 2006 [National unit cost report in Finnish health care 2006]. Työpapereita/Stakes: 3/2008. Helsinki, Finland: Stakes; 2008. Available from: http://urn.fi/URN:NBN:fi-fe201204194178. Accessed August 26, 2015. Finnish.

21. Krieckaert CL, Nair SC, Nurmohamed MT, et al. Personalised treatment using serum drug levels of adalimumab in patients with rheumatoid arthritis: an evaluation of costs and effects. Ann Rheum Dis. 2015;74(2):361-368.
Biologics: Targets \& Therapy

\section{Publish your work in this journal}

Biologics: Targets \& Therapy is an international, peer-reviewed journal focusing on the patho-physiological rationale for and clinical application of Biologic agents in the management of autoimmune diseases, cancers or other pathologies where a molecular target can be identified. This journal is indexed on PubMed Central, CAS, EMBase, Scopus

\section{Dovepress}

and the Elsevier Bibliographic databases. The manuscript management system is completely online and includes a very quick and fair peerreview system, which is all easy to use. Visit http://www.dovepress. $\mathrm{com} /$ testimonials.php to read real quotes from published authors. 